\title{
Variables neuropsicológicas en pacientes con trastornos de la conducta alimentaria
}

\author{
Beatriz Mata ${ }^{1}$, Rafael Baena ${ }^{2}$, Leonor Asensio ${ }^{3}$, Laura Nuevo ${ }^{1}$, \\ Victoria Muñoz ${ }^{1}$, e Irene Díaz ${ }^{1}$ \\ ${ }^{1}$ Hospital General Universitario de Ciudad Real (España); \\ ${ }^{2}$ Hospital Universitario Fundación Alcorcón (España); \\ ${ }^{3}$ Hospital Juan Ramón Jiménez, Huelva (España)
}

\begin{abstract}
Parecen existir alteraciones en algunas variables neuropsicológicas en pacientes con trastornos de conducta alimentaria (TCA). El objetivo fue analizar las dimensiones neuropsicológicas atención sostenida e inhibitoria, velocidad de procesamiento, funciones ejecutivas y memoria de trabajo en pacientes con TCA. Se reclutaron 63 pacientes que acudieron a consultas. La distribución por subtipos de TCA fue $33 \%$ bulimia nerviosa (BN) purgativa, 24\% anorexia nerviosa (AN) purgativa, 13\% AN restrictiva incompleta, $19 \%$ AN restrictiva, $11 \%$ trastorno por atracón, $5 \%$ AN purgativa incompleta, $5 \%$ BN purgativa incompleta, $2 \%$ BN no purgativa y $2 \%$ ingesta compulsiva. Se administraron los tests: Stroop, Letter Number Sequencing (LNS), Symbol Digit Modalities (SDMT) y Figura de Rey. En el LNS la media fue 18,03, SpanLN 4,87; la interferencia fue 4,4 de media; el baremo de SDMT dio como resultado bajo- normal de media; el tiempo de media de la copia de la figura de Rey fue 2,81, con un centil 45 cuantitativo de memoria. Según subtipos de TCA del Diagnostic and Statistical Manual of Mental Disorders parece existir una distribución similar entre trastornos, con desviaciones de la población de referencia en el SDMT, la interferencia y la puntuación cuantitativa de la reproducción de memoria de la figura de Rey.
\end{abstract}

Palabras clave: Trastornos de la conducta alimentaria, atención, funciones ejecutivas, anorexia nerviosa, bulimia nerviosa, trastorno por atracón.

Neuropsychological dimensions in patients with eating disorders. Some neuropsychological dimensions may be deteriorated in patients with Eating Disorders (ED). Our objective was to analyse these dimensions in patients with ED: attention and vigilance, speed of information processing, executive function and working memory. 63 patients were collected. The percentages of patients by subtypes of ED were: $33 \%$ bulimia nervosa (BN) binge eating/purging, $24 \%$ anorexia nervosa (AN) binge eating/purging, $13 \%$ incomplete $\mathrm{AN}$ restricting, $19 \% \mathrm{AN}$ restricting, $11 \%$ binge eating disorder, $5 \%$ incomplete AN binge eating/purging, 5\% incomplete $\mathrm{BN}$ binge eating/purging, $2 \% \mathrm{BN}$ non- binge eating/purging and $2 \%$ binging. The following tests were administrated: Stroop, Letter Number Sequencing (LNS), Symbol Digit Modalities (SDMT) and The Rey complex figure test (RCFT). Average score in LNS was 18.03, SpanLN 4.87; the average interference was 4.4; average SDMT (related to reference population) was low-normal; average time to copy RCFT was 2.81 , quantitative centile in memory 45. It seems to be similar distribution of scores between different Diagnostic and Statistical Manual of Mental Disorders subtypes of ED. SDMT score, interference and quantitative score of memory in ROCF were worse than reference population.

Keywords: Eating disorders, attention, executive functions, anorexia nervosa, bulimia nervosa, binge eating.

Correspondencia: Correspondencia: Beatriz Mata Saenz. Servicio de Psiquiatría Hospital General Universitario de Ciudad Real. Avd. Obispo Rafael Torija s/n. C.P.: 13005. Ciudad Real (España). E-mail: bmatas@sescam.jccm.es 
Los trastornos de la conducta alimentaria (TCA) han supuesto un motivo de preocupación social y mediática en las últimas décadas. Aun así, a pesar de que esta enfermedad preocupa por su prevalencia, se estima que continúan siendo muchos los casos que no llegan a detectarse ni a consultar. La globalización de los cánones de belleza y su importación a países no industrializados, otorgan a este fenómeno un carácter mundial (Solmi, Hotopf, Hatch, Treasure, y Micali, 2016).

A pesar de que en múltiples ocasiones se ha resaltado la existencia de TCA desde hace siglos (como los periodos de ayunas de las santas de la Edad Media o los episodios de bulimia de las bacanales de la Antigüedad), no es hasta 1888 que se describe el primer caso de anorexia nerviosa $(\mathrm{AN})$ en la revista The Lancet, identificándose otros casos similares entre los pacientes atendidos por los médicos lectores de la revista (Mackenzie, 1888).

Los criterios de AN han ido cambiando a lo largo del tiempo, especialmente en lo referente a la cantidad de peso mínimo considerado como patológico y a la necesidad de cumplir el criterio de ausencia de la menstruación (que no siempre define la gravedad de la pérdida de peso y no es aplicable en mujeres prepúberes, postmenopáusicas ni que tomen medicación anticonceptiva, ni los varones). Además, en la última revisión de los criterios, se tiende a la consideración de la persistencia de conductas que impiden la recuperación ponderal en lugar de enfocarse en el tipo de pensamientos que tiene el sujeto (Moskowitz y Weiselberg, 2017).

En la última edición del Diagnostic and Statistical Manual of Mental Disorders (DSM5) se ha procurado definir más exactamente los criterios del grupo anteriormente denominado "trastorno de la conducta alimentaria no especificado", que englobaba una serie muy variada de pacientes. Algunos de ellos, eran pacientes en los cuales se cumplían, por ejemplo, todos los criterios de una AN pero que partían de un peso premórbido elevado y por ello no tenían un peso bajo a pesar de una pérdida del mismo muy elevada y patológica (Moskowitz y Weiselberg, 2017).

En 1979, se describieron por primera vez los primeros 30 casos de bulimia nerviosa (BN) (Russel, 1979). Clásicamente se diferenciaba a estas pacientes de las que padecían AN por tener un peso mayor (normal o incluso ligeramente elevado), menos problemas con la menstruación y mayor activación a nivel sexual y niveles de depresión. No se diferenciaron como otra variedad de TCA, sino que se consideraba una forma evolutiva de la AN, con mayor riesgo de complicaciones por su escasa respuesta al tratamiento, complicaciones físicas y riesgo de suicidio. Esta última consideración pronóstica fue finalmente rechazada, considerándose que este trastorno tiene mejor pronóstico, aunque más tendencia a las recaídas, y que constituye una entidad diagnóstica distinta (Russell, 2004).

Los pacientes con BN suelen estar infradiagnosticados, ya que no despiertan tantas sospechas en el entorno sociofamiliar ni médico (su peso es, generalmente, normal, 
y sus menstruaciones se mantienen) y la ocultación de los síntomas de purga y atracones es muy frecuente, en parte por vergüenza y en parte por la falta de conciencia de enfermedad (Castillo y Weiselberg, 2017).

En el Diagnostic and Statistical Manual of Mental Disorders (DSM)-IV, se distinguieron dos subtipos de BN: purgativa (en la que existen conductas de purga como la toma de laxantes, enemas o diuréticos, o la provocación de vómitos) y no purgativa (en la cual es el ejercicio físico excesivo el que se emplea como conducta compensatoria); sin embargo, en la clasificación del DSM-5, desaparece esta distinción al no haberse observado diferencias evolutivas (a pesar de lo cual, en nuestro trabajo, incluimos esta separación por si pudiese influir de algún modo en los resultados). Además, la frecuencia necesaria de conductas de purga-atracón desciende de 2 a la semana durante 3 meses a una a la semana (Castillo y Weiselberg, 2017).

La existencia de un TCA se ha asociado con algunas alteraciones neuropsicológicas evaluadas mediante instrumentos de exploración, lo que podría tener implicaciones terapéuticas (Tchanturia et al., 2008, 2011, 2012). Sin embargo, no son muchos los estudios que valoran la relación entre los TCA y el funcionamiento cognitivo. En esta línea, se han encontrado ciertas deficiencias en la realización del test de la figura de Rey, que comprende la copia de imágenes visuales complejas y permite analizar el manejo del foco de los diferentes niveles de la imagen. En comparación con controles, los pacientes con TCA tienden a focalizar la atención en mayor grado en los detalles que en la idea global de la imagen. Por otro lado, parece existir una escasa coherencia central, que se refiere a la incapacidad relativa de estas personas a realizar un equilibrado procesamiento de los detalles y el contexto. Estos resultados se replican en diferentes trabajos, con un claro empeoramiento en las puntuaciones de la imagen de recuerdo diferido respecto controles en pacientes con BN (Darcy et al., 2015).

En otro estudio, (Moynihan, Rose, van Velzen, y de Fockert, 2016) administraron a los pacientes diagnosticados de TCA el Navon Test. En él, se presentan estímulos jerárquicos consistentes en muchos elementos locales que forman una figura global mayor. La tarea consiste en identificar un estímulo diana en el nivel local o global, mientras se ignora el otro nivel. Los participantes, típicamente tendían a mostrar una vía de procesamiento global, respondiendo más rápido a las dianas globales que a las locales, y tenían un efecto de compatibilidad menor cuando respondían a las dianas locales vs las globales. Se encontraron a nivel electroencefalográfico diferencias en componentes que apoyan estos hallazgos psicométricos.

Continuando con otras valoraciones neurocognitivas en esta población, algunos autores señalan el descenso de la flexibilidad cognitiva en pacientes con TCA en relación con controles sanos (toma de decisiones, adaptación a normas, resolución de problemas) (Galimberti et al., 2013; Tchanturia et al., 2012; Tenconi et al., 2010). En pacientes con $\mathrm{BN}$, se ha descrito la existencia de déficits en la atención, mayor impulsividad y 
empeoramiento de la flexibilidad cognitiva (Hirst et al., 2017). En estudios con familiares sanos, parece existir cierta reproducción de estos déficits, lo que plantea la posibilidad de que constituyan un marcador biológico (Galimberti et al., 2013; Van den Eynde et al., 2011).

Sin embargo, la naturaleza de estos hallazgos está discutida, existiendo autores que los señalan como parte de la etiopatogenia de los TCA y otros que plantean la influencia de la malnutrición como factor en el desarrollo de déficits cognitivos, especialmente en aquellos casos de TCA que se inician en la infancia o la adolescencia, así como la participación de un mayor coeficiente intelectual como factor de vulnerabilidad de desarrollo de un TCA (Schilder et al., 2017). Algunos de estos resultados son los existentes, por ejemplo, dentro del paradigma atencional, valorados con el test de Stroop (interferencia) (Golden, 1993) o el Test de símbolos y dígitos (Smith, 2002), cuyos déficits podrían relacionarse en TCA sobre todo con variables nutricionales.

Existen escasos estudios en nuestro medio sobre dimensiones neurocognitivas en pacientes con TCA, por lo que, como parte de un trabajo de mayor envergadura, planteamos como objetivo analizar las dimensiones neuropsicológicas atención sostenida e inhibitoria, velocidad de procesamiento, funciones ejecutivas y memoria de trabajo en pacientes con TCA, dada su probable importancia diagnóstica, clasificatoria y terapéutica.

\section{MÉTODO}

\section{Participantes}

Se consideraron como participantes todos aquéllos pacientes que acudan a la consulta de TCA durante el periodo designado (6 meses, desde enero a junio de 2018), cumplieron criterios de inclusión y no de exclusión y deseasen y consintiesen participar en el mismo.

La población de referencia de nuestras consultas de trastornos de la conducta alimentaria es la atendida en 19 áreas básicas de salud de nuestra provincia, Ciudad Real. Esto abarca una población de 165.976 usuarios.

La participación fue voluntaria sin que el rechazo en participar o la retirada del estudio constituyese un factor a tener en cuenta en la continuidad del tratamiento en nuestra Unidad. Del mismo modo, no se recibió gratificación por la participación.

\section{Criterios de inclusión}

Pacientes con criterios diagnósticos según DSM-5 de anorexia nerviosa, bulimia nerviosa, trastorno por atracón y trastorno de la conducta alimentaria no especificado, como diagnóstico principal atendidos en la Unidad de trastornos de la conducta alimentaria del Hospital General Universitario de Ciudad Real. 
- $\quad$ Sexo femenino

Edad entre 18 y 50 años.

Encontrarse físicamente capaces de participar en el estudio. Criterios de exclusión:

Dificultades idiomáticas que impidan entender los instrumentos de evaluación y las instrucciones de las terapias.

No firmar el consentimiento informado del estudio/no desear participar en el mismo.

- Sexo masculino

Diagnósticos comórbidos de trastorno afectivo bipolar, trastorno psicótico o trastorno depresivo mayor, daño cerebral confirmado, trastorno por déficit de atención, trastornos del espectro autista, síndrome de Tourette o síndrome de fatiga crónica.

De las 63 pacientes valoradas, la edad media fue de 29 años $(D T=9,1)$ y el IMC medio fue de 22,2 ( $D T=7,1)$. El $86 \%$ de las pacientes fueron derivadas de las consultas y el $14 \%$ restante, de la planta de Hospitalización. El nivel de estudios fue básico en un $35 \%$ de las pacientes (bachillerato sin terminar o inferior) y superior en el $65 \%$.

\section{Instrumentos y variables}

Sexo y edad: recogidas a partir de una entrevista semiestructurada.

Test de Secuencia de letras y números (Letter Number Sequencing, LNSIII): el test de Secuencia de Letras y Números (LNS) forma parte de la Wechsler Adult Intelligence Scale (WAIS)-III y IV. En este test se obtienen los siguientes índices: suma de intentos (que equivale al número de intentos correctamente contestados por el sujeto) y SpanLN (que corresponde con el número de dígitos máximo recordado por el sujeto). Está considerada como una de las subpruebas clásicas de memoria de trabajo, consiste en ofrecer verbalmente al examinado una secuencia creciente de letras y números que deberá retener, manipular (ordenar) y devolver al examinador. Los estudios normativos para la secuenciación de letras y números indican una influencia moderada de la edad, por lo que existen datos normativos para sujetos mayores de 50 años (Tamayo et al., 2012).

Test de Stroop de colores y palabras (Stroop, 1935): la interferencia se produce al activarse el proceso automático de respuesta verbal que interfiere con el nombramiento de los colores. Para realizar la tarea, la persona tendrá que lograr secuenciar ambas tareas (leer la palabra y después nombrar el color), lo cual hará que la velocidad sea lenta, o suprimir de forma voluntaria la lectura automática de la palabra. Algunas personas logran suprimir la lectura y centrarse únicamente en la tarea de nombrar los colores, otros no son capaces de hacerlo, por lo que tienen que leer la palabra primero y después nombrar el color, y otros, en los que el nivel de interferencia es alto, tendrán grandes dificultades para separar ambas respuestas (Golden, 1993). 
Test de símbolos y dígitos (Symbol Digit Modalities test, SDMT) (Smith, 1982): es una prueba que evalúa principalmente atención (incluyéndose dentro del paradigma de la atención sostenida), rastreo visual, velocidad de procesamiento mental y velocidad visomotora. El test consiste en emparejar dibujos geométricos sin significado con un número del 1 al 9, según un modelo previo. En nuestro trabajo, se empleará la versión del SDMT de Smith (2002).

Test de copia de una figura compleja (Rey Complex Figure Test, RCFT) (Rey, 1959): la tarea de esta prueba consiste en copiar primero y reproducir después, de memoria, una figura. Evalúa la presencia de posibles trastornos neurológicos y cognitivos relacionados con problemas de carácter perceptivo, motriz o de memoria, así como el grado de desarrollo de la actividad gráfica.

Peso, altura e índice de masa corporal (IMC) (Quetelet, 1836): Las participantes del estudio son talladas y pesadas por personal de enfermería entrenado para ello. El peso es medido por una báscula, con ropa y sin zapatos. La estatura se mide mediante un tallímetro de pared sin zapatos. El IMC es una medida de asociación entre la masa y la talla de un individuo que se calcula mediante la fórmula $\mathrm{IMC}=$ masa (en kilogramos) /talla (en metros) elevada al cuadrado (expresándose en la unidad kg/m2). La principal desventaja de este índice es que, al evaluarlo a nivel individual, puede dar una idea engañosa de la composición corporal, ya que, realmente, sólo relaciona peso con altura, sin especificar otros componentes no grasos de la masa corporal (Ministerio de Sanidad, Consumo y Bienestar Social, 2018). Se puede considerar el IMC distribuido en los siguientes 8 tramos: insuficiencia ponderal $(<18,5)$, intervalo normal $(18,5-24,9)$, sobrepeso $(>=25)$, preobesidad $(25-29,9)$, obesidad $(>=30)$, obesidad clase I $(30-34,9)$, clase II $(35-39,9)$ y clase III (>=40) (OMS, 2003).

Tiempo de evolución de la enfermedad; presencia o no de conductas purgativas y atracones; criterios de bulimia, anorexia nerviosa y trastorno por atracones (Asociación Americana de Psiquiatría, 2014); tratamiento farmacológico recibido.

\section{Procedimientos}

Para la realización del estudio, se contó con la aprobación del Comité Ético de Investigación Clínica del Hospital General Universitario de Ciudad Real.

Todos los diagnósticos fueron realizados por un especialista en Psiquiatría y corroborados con el equipo especialista en TCA que atendía a las pacientes. Así mismo, el estado clínico de las participantes fue consultado con ambos especialistas de las Unidad, en base a criterios de la DSM-5.

Antes de iniciar el estudio, las participantes recibieron información referente al mismo por escrito, cumplimentándose un consentimiento informado.

Las pacientes completaron una batería de tests neuropsicológicos reconocidos a nivel internacional con validaciones españolas cubriendo un espectro de diferentes 
dominios cognitivos que se habían visto posiblemente afectados en estudios previos. Los test fueron aplicados y corregidos por una psiquiatra formada en Neuropsicología. A pesar de que originalmente se les ofrecía realizar los test por la tarde (para evitar posibles distractores), por razones de comodidad para las participantes, la mayoría (68\%) fueron valoradas por la mañana antes de entrar a consulta con sus referentes.

El orden de realización de la valoración fue:

$1^{\mathrm{o}}$ : Acogida y lectura al paciente de la Hoja de Información al Paciente.

$2^{\circ}$ : Lectura y firma del consentimiento informado de participación.

$3^{\circ}$ : Administración del test LNS.

$4^{\text {o: }}$ : Administración del test de Stroop. $5^{\circ}$ : Administración del SDMT.

$6^{\text {o: }}$ Administración de la fase de copia de la Figura de Rey.

$7^{\circ}$ : Realización de la entrevista semiestructurada y administración del test de la Figura de Gardner (no incluida en este trabajo) (menos de 3 minutos).

$8^{\text {o: }}$ Realización de fase de memoria de la Figura de Rey. $9^{\text {o }}$ : Cierre de la entrevista.

\section{Análisis de datos}

Los datos obtenidos se analizaron con el programa SPSS 19.0. Todos los procedimientos estadísticos se resolverán utilizando un nivel de significación del 5\% (alfa $=0,05)$ e intervalos de confianza estadística del 95\%. Se analizará la existencia o no de normalidad de los datos de la muestra mediante los test de Kolmogorov-Smirnov (más de 50 datos) o Shapiro-Wilk (menos de 50 datos). Se estudiará, en primer lugar, la relación estadísticamente significativa o no de las variables clínicas con las neuropsicológicas y de ambas con los parámetros de la onda P300. En segundo lugar, se analizará la relación de estas variables con la evolución tras un año de tratamiento. Para el análisis estadístico se utilizarán pruebas no paramétricas (Mann-Whitney, Wilcoxon, y Kruskal-Wallis) y paramétricas ( $\mathrm{t}$-Student, pruebas ANOVA) para las variables dependientes cuantitativas $\mathrm{y}$, convenientemente, se utilizarán pruebas para variables dependientes categóricas (pruebas de independencia de Chi-cuadrado y de Fischer).

\section{RESULTADOS}

\section{Variables clínicas}

Se resumen los datos aportados de la sintomatología por las pacientes en la siguiente tabla (tabla 1) y gráfico (gráfico 1), refiriéndose a síntomas que se han presentado en alguna ocasión a lo largo de la evolución (no siempre presentes en el momento de la valoración). 
MATA et al. Variables neuropsicológicas en TCA

Tabla 1. Resumen de la sintomatología según las participantes del estudio (\%)

\begin{tabular}{lcc}
\hline Síntoma/conducta & Presente & Ausente \\
\hline Atracones & 76 & 24 \\
\hline Vómitos provocados & 70 & 30 \\
\hline Uso de laxantes & 36 & 64 \\
\hline Uso de pastillas adelgazantes & 22 & 78 \\
\hline Uso de diuréticos & 30 & 70 \\
\hline Práctica de ejercicio compensatorio & 76 & 24 \\
\hline Restricción de comidas & 94 & 6 \\
\hline
\end{tabular}

Gráfico 1. Resumen del número de pacientes que presentan cada uno de los síntomas

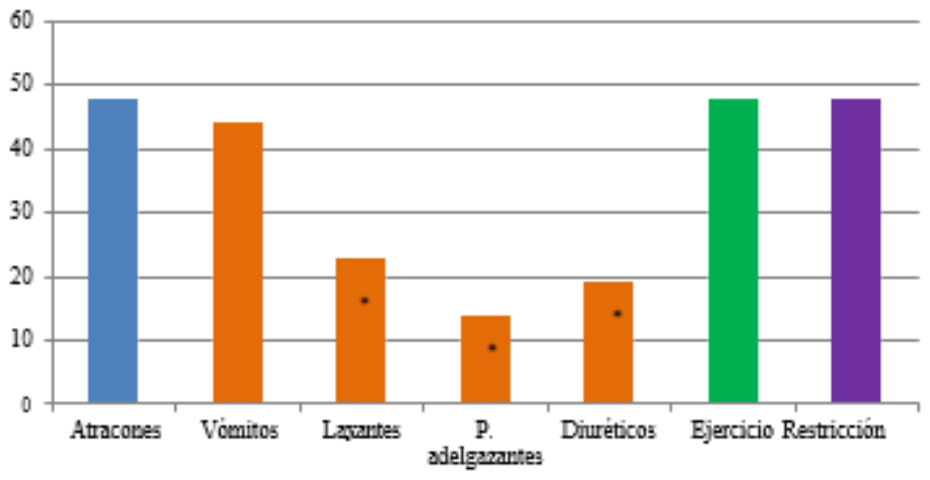

-Conductar purgativas

La distribución de los diagnósticos (seleccionando en los casos en los que se cumplían criterios para dos diagnósticos aquel que había presentado mayor duración y gravedad) fue (tabla 2 y gráfico 2 ).

Tabla 2. Resumen de diagnósticos

\begin{tabular}{llc}
\hline \multicolumn{1}{c}{$n$} & Porcentaje \\
\hline Anorexia nerviosa & 25 & 37 \\
\hline Anorexia nerviosa restrictiva & 11 & 17 \\
\hline Anorexia nerviosa purgativa & 14 & 22 \\
\hline Bulimia nerviosa & 18 & 28 \\
\hline Bulimia nerviosa purgativa & 16 & 25 \\
\hline Bulimia nerviosa no purgativa & 2 & 3 \\
\hline $\begin{array}{l}\text { Trastorno de la conducta } \\
\text { alimentaria no especificado }\end{array}$ & 13 & 18 \\
\hline $\begin{array}{l}\text { Anorexia nerviosa restrictiva } \\
\text { incompleta }\end{array}$ & 8 & 13 \\
\hline $\begin{array}{l}\text { Anorexia nerviosa purgativa } \\
\text { incompleta }\end{array}$ & 2 & 3 \\
\hline $\begin{array}{l}\text { Bulimia nerviosa purgativa } \\
\text { incompleta }\end{array}$ & 3 & 11 \\
\hline Trastorno por atracón & 7 & 5 \\
\hline
\end{tabular}


Gráfico 2. Distribución de los diagnósticos en la muestra

$$
\begin{aligned}
& \text { Anorexia } \\
& \text { nerviosa } \\
& \text { restrictiva }
\end{aligned}
$$

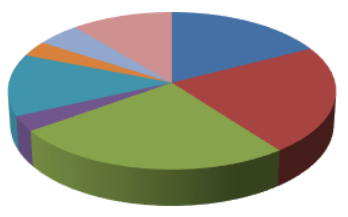

El 54\% de las pacientes se encontraban en tratamiento con algún psicofármaco. De estas pacientes, el $85 \%$ recibían tratamiento con antidepresivos, $61 \%$ con benzodiacepinas y $9 \%$ con antipsicóticos y anticomiciales, respectivamente. No parece existir ninguna relación (según prueba de Chi-cuadrado) estadísticamente significativa entre el uso o no de fármacos y los diagnósticos DSM-5, ni entre estos y el uso de uno u otro fármaco, excepto para los anticomiciales (que en todos los casos fueron empleados en pacientes con bulimia nerviosa) (gráficos 3 y 4).

Gráfico 3. Distribución del uso o no de fármacos en los diferentes diagnósticos

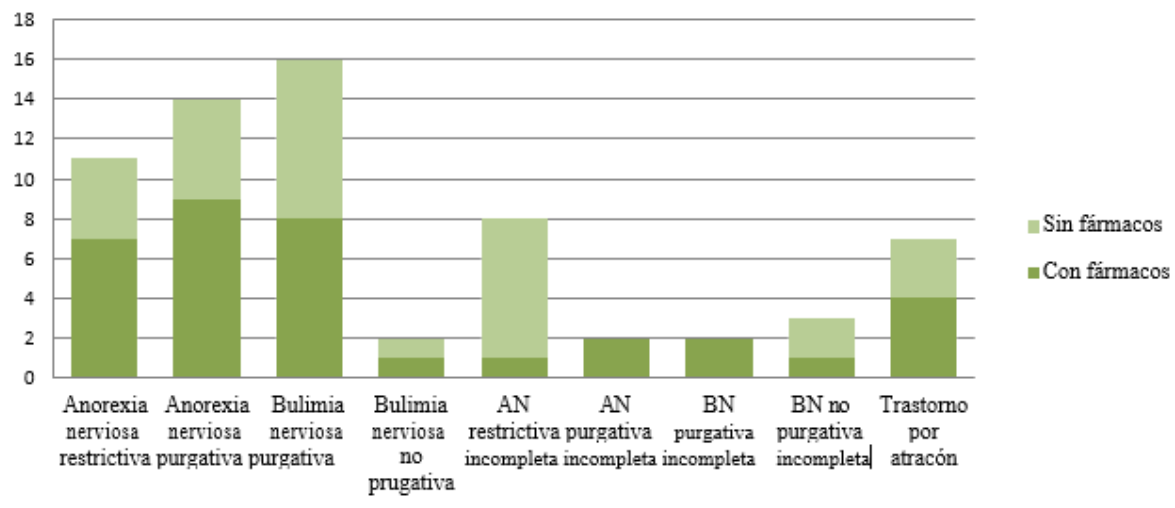


Gráfico 4. Distribución de los diferentes tipos de psicofármacos según diagnóstico

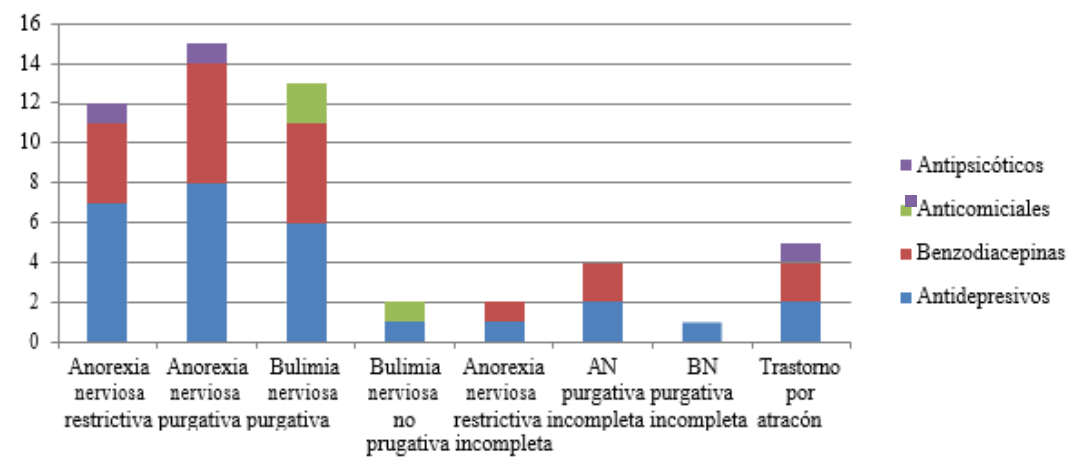

\section{Variables neuropsicológicas}

\section{Test LNS}

La media de la suma de intentos fue de $18(D T=2,6)$ y la del SpanLN 4,9 (DS 0,9). No se encontraron diferencias estadísticamente significativas por diagnóstico ni uso de psicofármacos.

\section{Test de Stroop}

El cálculo de la interferencia, resultó, de media 4,5 (DT=11,7). No se encontraron diferencias estadísticamente significativas por diagnóstico ni uso de psicofármacos.

SDMT:

La media de aciertos en el test fue de 53,9 (DT=11,7), sin diferencias entre diagnósticos ni uso de psicofármacos estadísticamente significativas.

Test de la figura de Rey:

El tiempo medio en la realización de la copia fue de 2,8 minutos $(D T=1,0)$. El de realización de la figura de memoria fue de 2,6 minutos $(D T=1,2)$, sin diferencias estadísticamente significativas con el uso de psicofármacos (T-Student para muestras independientes). Sin embargo, existen diferencias entre diagnósticos en el tiempo de ejecución del ejercicio de copia (ANOVA).

La puntuación media en la valoración cuantitativa de la copia fue de 34,4 $(D T=2,4)$ y de la memoria $19,7(D T=6,0)$. Ambas sin diferencias estadísticamente significativas entre diagnósticos ni uso de psicofármacos.

Al valorar los aspectos cualitativos de la realización de la figura se obtuvieron los siguientes resultados (gráfico 5). 
Gráfico 5. Distribución de los tipos de realización de la copia de la figura de Rey en la muestra

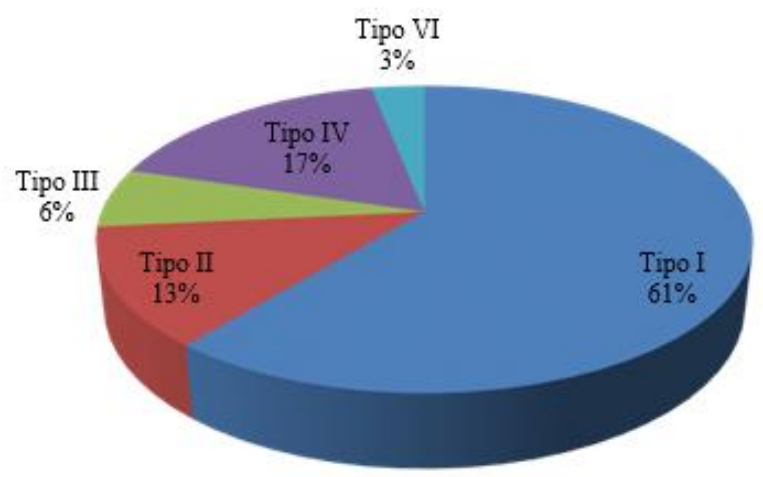

Tabla 3. Resumen de las variables neuropsicológicas

\begin{tabular}{lc}
\hline Test & Puntuación media (desviación típica) \\
\hline Test LNS & $18(2,6)$ \\
\hline Suma & $4,9(0,9)$ \\
\hline SpanLN & $4,5(11,7)$ \\
\hline Test de Stroop & \\
\hline Interferencia & $53,9(11,7)$ \\
\hline SDMT & $2,8(1,0)$ \\
\hline Aciertos & $34,4(2,4)$ \\
\hline Test de la Figura de Rey & $2,6(1,2)$ \\
\hline Puntuación copia & $19,7(6,0)$ \\
\hline Tiempo memoria &
\end{tabular}

No se hallaron diferencias estadísticamente significativas entre diagnósticos (prueba ANOVA) ni uso de psicofármacos (t-Student para muestras independientes) ni IMC (correlación lineal) y las puntuaciones de los test realizados.

\section{DISCUSIÓN/CONCLUSIONES}

\section{Test de Stroop}

El cálculo de la interferencia, resultó, de media 4,5 (DT=11,7), en el percentil 52 respecto a muestras españolas (en las cuales la media es de 2,7, $D T=8,53$ ). Dada la influencia por edad, se realizó la corrección precisa en los casos que lo requerían (Golden, 1993). 
En los estudios realizados no siempre se obtiene una mayor dificultad en completar tareas de medida del control inhibitorio. No obstante, los paradigmas empleados son muy diversos, variando desde las tareas de Stroop clásicos hasta variaciones del mismo. Además, no siempre se obtienen las medidas completas y estandarizadas ni se analiza el tamaño del efecto observado (Van den Eynde et al., 2011).

\section{SDMT}

La media de aciertos en el test fue de 53,9 (DT=11,7). Al comparar con población española (Smith, 2002), la puntuación obtenida de media se considera en el límite superior del rango considerado bajo, ajustando por estudios y edad. No parece existir influencia por IMC.

Aunque los estudios realizados en pacientes con $\mathrm{BN}$ analizando la atención han arrojado resultados contradictorios, los trabajos que han empleado el SDMT no han obtenido diferencias con controles sanos (Van den Eynde et al., 2011).

\section{Test de la figura de Rey}

El tiempo medio en la realización de la copia fue de 2,8 minutos $(D T=1,0)$, lo que estaría en el percentil 50 de la muestra española (Rey, 2009). El de realización de la figura de memoria fue de 2,6 minutos $(D T=1,2)$, siendo la mediana en adultos de 4 minutos en muestras españolas. La puntuación media en la valoración cuantitativa de la copia fue de 34,4 (DT=2,4) y de la memoria 19,7 $(D T=6,0)$. El centil en el que, de media, se encontraron las participantes en la realización de la copia (valoración cuantitativa) fue el centil 85, y la figura de memoria, 45, en relación con población española.

Al comparar con baremos en población española, el tipo I corresponde a un centil 75, el tipo II al centil 50 y el tipo III al centil 25. El resto de los tipos en población adulta normal no se describe. Por ello, podemos destacar el aumento de la proporción de sujetos que realizaron el tipo IV y VI en nuestra muestra.

En diferentes estudios, se ha señalado que quizás la menor tendencia a la coherencia central en el procesamiento de las imágenes pudiese ser un rasgo cognitivo endofenotípico de los TCA, diferenciando algunos autores una tendencia a la atención al detalle en los pacientes con AN en contraposición con una mala integración global en los que padecen BN (Darcy et al., 2015). Parece existir una mejoría de estos índices de coherencia central en pacientes con mejoría del peso y de los síntomas de enfermedad, aunque en nuestra muestra no se observa esa relación. Consideramos que la dificultad en la ejecución y organización de la Figura de Rey y la aparición de modelos más primitivos en el desarrollo cognitivo, así como la persistencia de esta manera de realización de la tarea en la reproducción de memoria, tiene relación estrecha con esta tendencia a la coherencia central y la mayor rigidez cognitiva (Galimberti et al., 2013; Hirst et al., 2017; Tchanturia et al., 2012; Tenconi et al., 2010). 
La importancia de estudiar variables como la afectación de la atención, la coherencia central o las funciones ejecutivas, radica en su influencia posible en los resultados terapéuticos. Así mismo, se ha barajado que pueda mejorarse, a través de su rehabilitación, la respuesta a terapias, especialmente de corte cognitivo-conductual (Tchanturia, 2008, 2011, 2012). En nuestro trabajo, analizando únicamente por diagnósticos DSM-5, parece existir un déficit claro en la función ejecutiva relativa a la coherencia central.

Como conclusión, se resaltan las alteraciones existentes a nivel ejecutivo, reflejadas en la realización de la Figura de Rey, que arroja alteraciones compatibles con las existentes en otros estudios en los que se destaca la dificultad para la integración global en pacientes con BN y la atención centrada en el detalle de las pacientes con AN. Estos hallazgos permiten entender el componente perceptivo-cognitivo que podría subyacer en la alteración de la imagen corporal y, con ello, en la base de los trastornos. Como limitaciones principales del estudio, se señalan el escaso número de participantes y la necesidad de control de otras variables de confusión psicopatológicas (especialmente ansiedad y depresión). Por ello, se plantea para líneas futuras la administración de cuestionarios psicopatológicos afectivos y alimentarios que permitan, así mismo, establecer relaciones transdiagnósticas de las variables neuropsicológicas con dimensiones psicopatológicas de los trastornos.

\section{REFERENCIAS}

Asociación Americana de Psiquiatría (2014). Manual diagnóstico y estadístico de los trastornos mentales. Madrid: Editorial Médica Panamericana.

Castillo, M., y Weiselberg, E. (2017). Bulimia nervosa/purging disorder. Current Problems in Pediatric and Adolescent Health Care, 47, 85-94.

Darcy, A.M., Fitzpatrick, K.K., Manasse, S.M., Datta, N., Klabunde, M., Colborn, D., ... Lock, J. (2015). Central coherence in adolescents with bulimia nervosa spectrum eating disorders. International Journal of Eating Disorders, 48(5), 487-493.

Galimberti, E., Fadda, E., Cavallini, M.C., Martoni, R.M., Erzegovesi, S., y Bellodi, L. (2013). Executive functioning in anorexia nervosa patients and their unaffected relatives. Psychiatry Research, 208, 238-244.

Golden, C.J. (1993). Stroop. Test de colores y palabras. Madrid: Ediciones TEA.

Hirst, R.B., Beard, C.L., Colby, K.A., Quittner, Z., Mills, B.M., y Lavender, J.M. (2017). Anorexia nervosa and bulimia nervosa: A meta-analysis of executive functioning. Neuroscience and Biobehavioral Reviews, 83, 678-690.

Mackenzie, S. (1888). On a case of anorexia nervosa vel hysterica. The Lancet, 131(3370), 613-614. Ministerio de Sanidad, Consumo y Bienestar Social (2018). La actividad física, la condición física y la salud infantojuvenil. Recuperado de: http://www.msssi.gob.es/ciudadanos/ proteccionSalud/adultos/actiFisica/ docs/capitulo2_Es.pdf.

Moskowitz, L., y Weiselberg, E. (2017). Anorexia nervosa/Atypical anorexia nervosa. Current Problems in Pediatric and Adolescent Health Care, 47, 70-84.

Moynihan, J., Rose, M., van Velzen, J., y de Fockert, J (2016). Local and global visual processing 
and eating disorder traits: An event-related potential study. Biological Psychology, 115, 27-34.

Organización Mundial de la Salud (OMS). (2003). Obesidad. Recuperado de: http://www.who.int/features/factfiles/obesity/facts/es/.

Quetelet, A. (1836). Sur l'homme et le developpement de ses facultes ou essai de physique sociale. Bruselas: Louis Hauman et comp.

Rey, A. (1959). Test de copie et reproduction de mémoire de figures géométriques complexes. París: Les Editions du Centre de Psychologie Appliquée.

Rey, A. (2009). Rey. Test de la copia y de reproducción de memoria de figuras geométricas complejas. Madrid: Ediciones TEA.

Russel, G. (1979). Bulimia nervosa: an ominous variant of anorexia nervosa. Psychological Medicine, 9(3), 429-448.

Russell, G.F.M. (2004). Thoughts on the 25th anniversary of bulimia nervosa. European Eating Disorders Review, 12(3), 139-152.

Schilder, C.M.T., van Elburg, A.A., Snellen, W.M., Sternheim, L.C., Hoek, H.W., y Danner, U.N. (2017). Intellectual functioning of adolescent and adult patients with eating disorders. International Journal of Eating Disorders, 50, 481-489.

Smith, A. (1982). Symbol Digit Modalities Test (SDMT). Manual (Revised). Los Angeles: Western Psychological Services.

Smith, A. (2002). Test de Símbolos y Dígitos. Madrid: Ediciones TEA.

Solmi, F., Hotopf, M., Hatch, S.L., Treasure, J., y Micali, N. (2016). Eating disorders in a multiethnic inner-city UK sample: prevalence, comorbidity and service use. Social Psychiatry and Psychiatric Epidemiology, 51(3), 369-381.

Stroop, J.R. (1935). Studies of interference in serial verbal reactions. Journal of Experimental Psychology, 18, 643-662.

Svaldi, J., Tuschen-Caffier, B., Biehl, S.C., Gschwendtner, K., Wolz, I., y Naumann, E. (2015). Effects of two cognitive regulation strategies on the processing of food cues in high restrained eaters. An event-related potential study. Appetite, 92, 269-277.

Tamayo, F., Casals-Coll, M., Sánchez-Benavides, G., Quintana, M., Manero, R.M., Rognoni, T.,... Peña-Casanova, J (2012). Estudios normativos españoles en población adulta joven (Proyecto NEURONORMA jóvenes): normas para las pruebas span verbal, span visuoespacial, Letter-Number Sequencing, Trail Making Test y Symbol Digit Modalities Test. Neurología, 27(6), 319-329.

Tchanturia, K., Davies, H., López, C., Schmidt, U., Treasure, J., y Wykes, T. (2008). Neuropsychological task performance before and after cognitive remediation in anorexia nervosa: a pilot case-series. Psychological Medicine, 38, 1371-1373.

Tchanturia, K., y Lock, J. (2011). Cognitive remediation therapy for eating disorders: development, refinement and future directions. Current Topics in Behavioral Neurosciences, 6, 269287.

Tchanturia, K., Davies, H., Roberts, M., Harrison, A., Nakazato, M.,... Morris, R. (2012). Poor cognitive flexibility in eating disorders: examining the evidence using the Wisconsin Card Sorting Task. Plos ONE, 7, 1, e28331.

Tenconi, E., Santonastaso, P., Degortes, D., Bosello, R., Titton, F., Mapelli, D., y Favaro, A. (2010). Set-shifting abilities, central coherence, and handedness in anorexia nervosa patients, their unaffected siblings and healthy controls: Exploring putative endophenotypes. The World Journal of Biological Psychiatry, 11, 813-823. 
Van den Eynde, F., Guillaume, S., Broadbent, H., Stahl, D., Campbell, I.C.,... Tchanturia, K. (2011). Neurocognition in bulimic eating disorders: A systematic review. Acta Psychiatrica Scandinavica, 124(2), 120-140.

Watts, K., Cranney, J., y Gleitzman, M. (2008). Automatic evaluation of body-related images. Body Image, 5(4), 352-364.

Recibido: 10 de diciembre de 2019

Recepción Modificaciones: 08 de enero de 2020

Aceptado: 10 de enero de 2020 